Annuaire suisse de politique de développement

$19 \mid 2000$

Pour une politique plus cohérente envers les pays en développement

\title{
2. Commerce mondial
}

Antoine Brawand

\section{(2) OpenEdition}

Journals

Édition électronique

URL : http://journals.openedition.org/aspd/848

DOI : $10.4000 /$ aspd. 848

ISSN : 1663-9669

Éditeur

Institut de hautes études internationales et du développement

Édition imprimée

Date de publication : 1 avril 2000

Pagination : 153-175

ISSN : 1660-5934

Référence électronique

Antoine Brawand, «2. Commerce mondial », Annuaire suisse de politique de développement [En ligne], 19 | 2000, mis en ligne le 18 août 2012, consulté le 08 septembre 2020. URL : http:// journals.openedition.org/aspd/848; DOI : https://doi.org/10.4000/aspd.848 


\section{COMMERCE MONDIAL *}

A CRISE FINANCIÈRE ASIATIQUE a entraîné un ralentissement général de l'activité économique et des échanges en 1998. Les pays exportateurs de matières premières ont subi le plus grand choc. Depuis 1999, l'économie mondiale bénéficie au contraire de la reprise observée en Asie du Sud-Est.

Deux faits majeurs ont marqué l'action de l'OMC: le changement de directeur général, intervenu après d'intenses tractations, et l'échec de la Conférence ministérielle de Seattle. Celle-ci aurait dû adopter le programme d'un nouveau cycle de négociations commerciales multilatérales. La Suisse s'était prononcée en faveur d'un programme étendu favorable aux compromis. Le blocage de Seattle n'empêche pas l'ouverture des négociations prévues depuis longtemps sur l'agriculture et les services, mais il les rend plus ardues.

Dans ce climat conflictuel, la CNUCED a préparé sa $10^{\circ}$ session quadriennale de Bangkok. Celle-ci offre une chance aux partisans du dialogue.

\section{1. ÉVOLUTION DU COMMERCE MONDIAL}

Croissance en volume de la production et des exportations mondiales de marchandises

En 1998, la production mondiale de marchandises a augmenté de 1,5\% en termes réels. En recul de 3 points par rapport à l'année précédente, ce taux marque un ralentissement de l'expansion économique (voir tableau 10). L'OMC impute ce freinage à la crise financière asiatique de 1997, dont l'impact mondial s'est exercé avec un certain décalage. Au niveau du commerce mondial, le ralentissement s'est traduit par une chute du taux de croissance en volume des exportations de marchandises, de 10,5\% en 1997 à $4 \%$ en 1998. Ce recul est intervenu après quatre années d'expansion très rapide. L'OMC note toutefois une similitude avec les résultats des années précédentes: la croissance du commerce mondial a, une fois de plus, dépassé celle de la production. Par rapport à 1990, la hausse annuelle moyenne des exportations de marchandises a atteint $6,5 \%$, alors que la production n'avait progressé que de $2 \%$. En conséquence, la part du commerce dans l'activité économique mondiale a continué d'augmenter'.

Tableau 10: Croissance en volume de la production* et des exportations mondiales de marchandises, 1990-98 (variations annuelles en pourcentage)

\begin{tabular}{lrrrr}
\hline & $\mathbf{1 9 9 0 - 9 8}$ & $\mathbf{1 9 9 6}$ & $\mathbf{1 9 9 7}$ & $\mathbf{1 9 9 8}$ \\
\hline Production de marchandises & 2.0 & 3.0 & 4.5 & 1.5 \\
\hline Exportation de marchandises & 6.5 & 6.0 & 10.5 & 4.0 \\
\hline
\end{tabular}

Source: OMC, Rapport annuel 1999, vol. 1, tableau II.1.

* La production mondiale de marchandises ne comprend ni la construction, ni les services. Cet agrégat diffère donc du PIB mondial.

* Par Antoine Brawand, chargé de cours à l'IUED.

1. OMC, Rapport annuel 1999, vol. 1, pp. 10-12. 
En 1999, la reprise économique en Asie du Sud-Est et la poursuite de l'expansion aux Etats-Unis ont entraîné une augmentation du taux de croissance de la production mondiale. L'OMC affirme que l'application des règles multilatérales a permis de maintenir l'ouverture des marchés et a ainsi contribué de façon décisive à la reprise ${ }^{2}$.

\section{Commerce mondial des biens et des services commerciaux}

La valeur des exportations mondiales de marchandises a atteint 5270 milliards de dollars en 1998, soit $2 \%$ de moins qu'en 1997 (voir tableau 11). Selon le rapport de l'OMC, ce recul a résulté d'une baisse des prix moyens d'environ $6 \%$ due essentiellement à la chute des cours des produits primaires (du pétrole en particulier), ainsi qu'à la poursuite de l'appréciation du dollar jusqu'au milieu de l'année ${ }^{3}$.

La valeur des exportations mondiales de services commerciaux a stagné en 1998 au niveau atteint l'année précédente, à environ 1300 milliards de dollars. Selon des données réunies par l'OMC, les prix moyens des services n'ont pas beaucoup varié. Les baisses des coûts de transport ont été compensées par des hausses modérées dans d'autres catégories de services. Par conséquent, le volume des échanges de services commerciaux est lui aussi resté inchangé, contrairement à celui du commerce des marchandises ${ }^{4}$ qui a augmenté de $4 \%$, comme mentionné plus haut. Par rapport à 1990, la valeur des exportations de services a augmenté annuellement de $7 \%$, soit un peu plus que celle des marchandises.

Tableau 11 : Croissance en valeur des exportations mondiales de marchandises et de services commerciaux (en milliards de dollars et en pourcentages)*

\begin{tabular}{lcrrrr}
\hline & $\begin{array}{c}\text { Valeurs } \\
\text { (milliards de dollars) }\end{array}$ & \multicolumn{3}{c}{$\begin{array}{c}\text { Variations annuelles } \\
\text { (pourcentages) }\end{array}$} \\
& $\mathbf{1 9 9 8}$ & $\mathbf{1 9 9 0 - 9 8}$ & $\mathbf{1 9 9 6}$ & $\mathbf{1 9 9 7}$ & $\mathbf{1 9 9 8}$ \\
\hline Marchandises & 5270 & 6.0 & 5.5 & 3.5 & -2.0 \\
\hline Services commerciaux & 1320 & 7.0 & 7.0 & 4.0 & 0.0 \\
\hline
\end{tabular}

Source: OMC, Rapport annuel 1999, vol. 1, tableau II.2 et II.5.

* Les données relatives aux exportations de services commerciaux et aux exportations de marchandises ne sont pas entièrement comparables, essentiellement parce que les premières sont tirées des statistiques de la balance des paiements et les secondes des statistiques douanières.

\section{$\square$ Commerce mondial des marchandises par régions}

En 1998, le volume des importations des pays d'Asie les plus affectés par la crise financière (Corée, Indonésie, Malaisie, Philippines et Thaïlande) s'est contracté de plus d'un cinquième. Par contre, le volume de leurs exportations a augmenté de $10 \%$. Quant aux importations japonaises, elles ont baissé de 5,5\% et les exportations de $1,5 \%$.

Les baisses de prix ont amplifié (ou atténué, selon le cas) les variations exprimées en valeur (voir tableau 12). Les importations des pays d'Asie les plus touchés par la crise ont chuté de $31 \%$ et leurs exportations de $4 \%$ (malgré une augmentation en volume de $10 \%$ ). La valeur des importations japonaises a baissé

2. Communiqué de presse, 17.11.99, pp. 1 et 5; Focus OMC, novembre 1999, pp. 5-6.

3. OMC, Rapport annuel 1999, vol. 1, pp. 8, 10 et 12 .

4. Ibid., p. 12.

5. Ibid., tableau II.3. 
de $17 \%$ et celle des exportations de $8 \%$. Les pays pétroliers ont subi une chute de leurs recettes d'exportation, en particulier ceux du Moyen-Orient (-22\%) et d'Afrique $(-31 \%)^{6}$.

Tableau 12: Croissance en valeur du commerce mondial des marchandises par régions, 1990-1998 (en milliards de dollars et en pourcentages)

\begin{tabular}{|c|c|c|c|c|c|c|}
\hline \multicolumn{3}{|c|}{ Exportations (f.à b.) ${ }^{a}$} & & \multicolumn{3}{|c|}{ Importations (c.a.f.) } \\
\hline $\begin{array}{l}\text { Valeur } \\
1998\end{array}$ & $\begin{array}{r}\% \\
1990-98\end{array}$ & $\begin{array}{r}\% \\
1998\end{array}$ & & $\begin{array}{r}\text { Valeur } \\
1998\end{array}$ & $\begin{array}{r}\% \\
1990-98\end{array}$ & $\begin{array}{r}\% \\
1998\end{array}$ \\
\hline 5270 & 5.7 & -1.9 & Monde entier & 5465 & 5.9 & -1.3 \\
\hline 897 & 7.0 & -0.7 & Amérique du Nord ${ }^{c}$ & 1152 & 7.6 & 4.6 \\
\hline 276 & 8.3 & -1.5 & Amérique latine & 340 & 13.7 & 5.1 \\
\hline 2348 & 4.6 & 2.9 & Europe occidentale $^{d}$ & 2367 & 4.2 & 4.9 \\
\hline 214 & 7.1 & -4.7 & Economies en transition $^{e}$ & 242 & 6.2 & -1.8 \\
\hline 107 & 0.5 & -15.2 & Afrique & 134 & 4.7 & 2.6 \\
\hline 137 & 0.3 & -22.2 & Moyen-Orient & 144 & 4.7 & -4.6 \\
\hline 1293 & 7.2 & -6.2 & Asie & 1086 & 5.5 & -17.8 \\
\hline 388 & 3.8 & -7.8 & Japon & 280 & 2.2 & -17.2 \\
\hline 184 & 14.5 & 0.5 & Chine & 140 & 12.8 & -1.5 \\
\hline 337 & 10.5 & -3.9 & $\begin{array}{l}\text { Pays les plus touchés } \\
\text { par la crise financière }\end{array}$ & 254 & 5.3 & -30.8 \\
\hline
\end{tabular}

Source: OMC, Rapport annuel 1999, vol. 1, tableau II.4.

${ }^{a}$ f.à b. : franco à bord.

b c.a.f. : coût, assurance, frais.

${ }^{\mathrm{c}}$ Canada et Etats-Unis.

'Les pays de I'Union européenne ont réalisé 2181 milliards de dollars d'exportations et 2172 milliards d'importations. Abstraction faite des échanges entre pays membres, les exportations de l'Union européenne ont atteint 813 milliards de dollars et les importations 801 milliards.

${ }^{e}$ Europe centrale et orientale, Etats baltes et Communauté d'Etats indépendants.

${ }^{\dagger}$ Corée, Indonésie, Malaisie, Philippines et Thaïlande.

\section{$\square$ Commerce mondial des marchandises par produits}

La baisse de $2 \%$ en valeur du commerce mondial des marchandises en 1998 provient de la chute des exportations de produits primaires. Le tableau 13 indique une diminution de $25 \%$ des exportations de combustibles et de $10 \%$ des exportations de matières premières agricoles. Ces baisses en valeur ont résulté des chutes de prix consécutives à l'augmentation de l'offre et à la faiblesse de la demande sur les marchés mondiaux. Comme mentionné plus haut, cette évolution défavorable aux pays en développement s'explique par le ralentissement de l'expansion économique entraîné par la crise asiatique.

Au cours de la même période, la valeur des exportations de produits manufacturés a légèrement augmenté. Les exportations de machines et de matériels de transport ont connu la plus grande progression, en particulier celles des produits de l'industrie automobile. Par contre, les exportations de textiles, de vêtements, de fer et d'acier ont diminué. Il s'agit, là aussi, de produits exportés dans une large mesure par des pays en développement.

L'évolution des exportations en 1998 a marqué une rupture avec les hausses enregistrées depuis 1990. Cependant, ces hausses elles-mêmes étaient grevées de disparités: les exportations de produits manufacturés avaient augmenté de $7 \%$, mais celles de produits agricoles seulement de $4 \%$ et les exportations des industries extractives (pétrole surtout) avaient stagné.

6. Principaux exportateurs africains de combustibles: Angola, Congo, Gabon, Lybie et Nigeria. 
La part des produits manufacturés dépasse maintenant les trois quarts des exportations mondiales, alors que les parts des produits agricoles et des produits des industries extractives sont tombées à un dixième chacune.

Tableau 13: Croissance en valeur des exportations mondiales de marchandises, par groupes de produits, 1990-98 (en milliards de dollars et en pourcentages)

\begin{tabular}{lrrrrr}
\hline & Valeurs & Parts & Variations annuelles \\
\hline & $\mathbf{1 9 9 8}$ & $\mathbf{1 9 9 0}$ & $\mathbf{1 9 9 8}$ & $\mathbf{1 9 9 0 - 9 8}$ & $\mathbf{1 9 9 8}$ \\
\hline Ensemble des produits* & $\mathbf{5 2 7 0}$ & $\mathbf{1 0 0 , 0}$ & $\mathbf{1 0 0 , 0}$ & $\mathbf{6}$ & $\mathbf{- 2}$ \\
\hline Produits agricoles & 553 & 12.2 & 10.5 & 4 & -5 \\
\hline Produits alimentaires & 443 & 9.3 & 8.4 & 4 & -4 \\
\hline Matières premières & 110 & 2.9 & 2.1 & 1 & -10 \\
\hline Produits des industries extractives & 502 & 14.3 & 9.5 & 0 & -20 \\
\hline Minerais et autres minéraux & 58 & 1.6 & 1.1 & 1 & -9 \\
\hline Combustibles & 344 & 10.5 & 6.5 & 0 & -25 \\
\hline Métaux non ferreux & 100 & 2.1 & 1.9 & 4 & -7 \\
\hline Produits manufacturés & 4010 & 70.6 & 76.1 & 7 & 1 \\
\hline Fer et acier & 141 & 3.1 & 2.7 & 4 & -2 \\
\hline Produits chimiques & 503 & 8.7 & 9.5 & 7 & 0 \\
\hline Autres produits semi-manufacturés & 399 & 7.8 & 7.6 & 5 & -1 \\
\hline Machines et matériel de transport & 2166 & 35.8 & 41.1 & 8 & 2 \\
\hline Textiles & 151 & 3.1 & 2.9 & 5 & -5 \\
\hline Vêtements & 180 & 3.2 & 3.4 & 7 & -1 \\
\hline Autres biens de consommation & 471 & 8.9 & 8.9 & 6 & 0 \\
\hline
\end{tabular}

Source: OMC, Rapport annuel 1999, vol. 2, tableau IV.1

* Y compris les produits non spécifiés (4\% du total en 1998).

\subsection{ACTIVITÉS COURANTES DE L'ORGANISATION MONDIALE DU COMMERCE (OMC)}

\section{$\square$ Objectifs, fonctions et budget de l'OMC}

L'OMC est le fondement juridique et institutionnel du système commercial multilatéral. Elle énonce les principales obligations contractuelles en fonction desquelles les gouvernements élaborent et mettent en œuvre leurs législations et réglementations nationales relatives au commerce. Elle constitue aussi la plateforme sur laquelle les relations commerciales entre les pays évoluent par le biais de négociations, de décisions et de débats collectifs.

Les fonctions essentielles de l'OMC sont les suivantes:

๖ administrer et mettre en œuvre les accords commerciaux multilatéraux et plurilatéraux qui, ensemble, constituent les règles de l'OMC;

๑ offrir un cadre aux négociations commerciales multilatérales ;

- s'efforcer de résoudre les différends commerciaux;

- examiner les politiques commerciales nationales;

- coopérer avec les autres institutions internationales qui s'occupent de l'élaboration des politiques économiques au niveau mondial.

Le Secrétariat de l'OMC, dont le directeur général est actuellement Mike Moore, emploie un peu plus de 500 fonctionnaires. Le budget ordinaire de

7. OMC, Rapport annuel 1999, vol. 1, pp. 42 et 113. 
l'OMC est passé de 122 millions de francs suisses en 1999 à 125 millions en 2000. Il est alimenté par les participations des pays membres au prorata de leur part dans le commerce international. Quant à la contribution annuelle de la Suisse, elle est passée de 2 millions de francs en 1999 (1,7\% du budget ordinaire de l'OMC) à 2,2 millions en 2000 ( $1,8 \%$ du budget $)$.

\section{$\square$ Une lutte de succession qui s'achève par un compromis}

Le mandat de Renato Ruggiero a pris fin en avril 1999. La procédure de nomination de son successeur débuta en automne 1998, mais les consultations révélèrent rapidement l'opposition entre deux camps de force relativement égale. Les Etats-Unis, l'Amérique latine et la plupart des pays européens soutenaient la candidature du Néo-Zélandais Mike Moore, tandis que l'Angleterre, la majorité des pays asiatiques et un bon nombre de pays africains favorisaient le Thaïlandais Supachai Panitchpakdi ${ }^{8}$.

Les deux hommes ont des passés différents, mais leurs objectifs se ressemblent. Mike Moore véhicule l'image d'un self-made-man ambitieux. Ancien premier ministre travailliste, il a toutefois laissé l'empreinte d'un ardent promoteur du libre-échange. Supachai Panitchpakdi est titulaire d'un doctorat en planification économique de l'Université Erasmus de Rotterdam. Il a fait une grande partie de sa carrière au sein de la banque centrale thaïlandaise. Il est devenu vicepremier ministre et ministre du Commerce avant tout pour ses compétences techniques. Les deux hommes ont représenté leurs pays aux négociations commerciales multilatérales du Cycle d'Uruguay. Ils estiment que leur origine leur facilitera l'établissement de ponts entre les pays riches et les pays pauvres. Ils promettent d'accélérer le processus d'accession à l'OMC, de rapprocher celle-ci de tous ses membres et de mieux communiquer avec la société civile ${ }^{9}$.

La règle du consensus ne permit pas de départager les deux candidats. Aucun n'acceptant de se retirer, le Conseil général, organe exécutif de l'OMC, décida de scinder le mandat de directeur général en deux périodes de trois ans chacune. Il nomma Mike Moore à partir du $1^{\text {er }}$ septembre 1999. Supachai Panitchpakdi entrera en fonction le $1^{\text {er }}$ septembre 2002. Les mandats ne pourront en aucun cas être renouvelés ou prolongés ${ }^{10}$.

Un élément du compromis était que Mike Moore présiderait le lancement du nouveau cycle de négociations commerciales multilatérales tandis que Supachai Panitchpakdi le bouclerait. La Suisse accueillit avec satisfaction la fin de la crise à l'OMC. Selon Pierre-Louis Girard, délégué aux accords commerciaux (Seco), le partage du mandat constituait néanmoins «une solution de seconde main ». La Suisse s'est montrée discrète dans le processus de nomination. L'ambassadeur suisse William Rossier avait en effet été chargé de jouer le rôle de facilitateur aux côtés du président du Conseil général, le Tanzanien Ali Mchumo" ${ }^{11}$.

Après de nouvelles consultations intensives, Mike Moore nomma quatre adjoints pour la durée de son mandat: Ablassé Ouedraogo (originaire du Burkina Faso),

8. «L'idée d'un partage de mandat à la tête de l'OMC gagne du terrain », Le Temps, 16.7.99.

9. «Désignation du prochain directeur général ", Focus OMC, juillet-août 1999, p. 2 ; «Un fauteuil pour deux directeurs: l'OMC sort de l'impasse", Le Temps, 21.7.99.

10. «Les gouvernements membres de l'OMC se mettent d'accord sur la succession du directeur général », Communiqué de presse, $\mathrm{n}^{\circ}$ 131, OMC, Genève, 22.7.99; «Un Néo-Zélandais, M. Mike Moore, à la tête de l'OMC », Focus OMC, juillet-août 1999, pp. 1-2.

11. «L'OMC reconnaît ses deux directeurs », Agence Télégraphique Suisse, citée par La Côte (Nyon), 23.7.99. 
Paul-Henri Ravier (France), Miguel Rodriguez Mendoza (Venezuela) et Andrew Stoler (Etats-Unis) ${ }^{12}$.

\section{$\square$ Pays membres et nouvelles accessions}

Depuis le $1^{\text {er }}$ janvier 1995, l'OMC a reçu huit nouveaux membres: l'Equateur, la Kirghizie, la Mongolie, la Lettonie et le Panama, ainsi que l'Estonie, la Géorgie et la Jordanie (les candidatures de ces trois derniers pays ont été acceptées en 1999). L'OMC compte actuellement 137 membres. Ensemble, ils réalisent un peu plus de $90 \%$ du commerce mondial.

Une trentaine d'autres pays ont déposé leur candidature et négocient leurs conditions d'accession ${ }^{13}$. Il s'agit surtout de pays en transition ou en développement. Quatre d'entre eux jouent un rôle de premier plan sur la scène économique et commerciale: la Chine ( $9^{\mathrm{e}}$ exportateur mondial en 1998), le «territoire douanier distinct» de Taiwan $\left(15^{\mathrm{e}} \mathrm{rang}\right)$, la Fédération de Russie $\left(19^{\mathrm{e}}\right)$ et l'Arabie Saoudite $\left(29^{\mathrm{e}}\right)^{14}$.

Tout Etat ou «territoire douanier» jouissant d'une entière autonomie dans la conduite de sa politique commerciale peut adhérer à l'OMC («accéder», dans la terminologie officielle), aux conditions convenues avec les membres de l'organisation. Les négociations sont souvent très complexes ${ }^{15}$. Le Conseil général de l'OMC nomme d'abord un groupe de travail. Celui-ci définit les conditions d'accession d'entente avec le candidat. Simultanément, le candidat et les membres intéressés de l'OMC mènent des négociations bilatérales concernant notamment l'accès au marché des biens et des services. (Les concessions faites par le candidat bénéficient à tous les membres de l'OMC conformément à la clause de la nation la plus favorisée.) Enfin, le groupe de travail soumet au Conseil général son rapport, un projet de «protocole d'accession» du futur membre, ainsi que les «listes» de concessions tarifaires et d'engagements relatifs aux services.

La candidature de la Chine $^{16}$ a entraîné l'ouverture de la négociation la plus longue. Des pays développés accusent la Chine de protectionnisme, mais veulent se réserver la possibilité de prendre des mesures de sauvegarde en cas d'afflux brusque de produits chinois sur leurs propres marchés. Le président du groupe de travail est actuellement l'ambassadeur Pierre-Louis Girard, délégué du Conseil fédéral aux accords commerciaux (Seco).

En novembre 1999, la Chine a enfin obtenu l'agrément du gouvernement américain. L'accord doit encore être approuvé par les instances législatives des deux pays. La Chine doit aussi s'entendre avec les autres membres de l'OMC. Les négociations avec l'Union européenne et avec le Canada seront décisives ${ }^{17}$. Mike Moore, directeur général de l'OMC, a déclaré à ce sujet: «C'est un grand pas en avant [...] J'ai dit à de nombreuses reprises que nous n'étions pas une

12. «La nomination de quatre adjoints: une longue bataille politique», Le Temps, 5.11.99

13. «Les accessions à l'OMC s'accélèrent», Focus OMC, octobre 1998, pp. 1-6. Information mise à jour en décembre 1999.

14. OMC, Rapport annuel 1999, vol. 2, tableau I.5.

15. «Dans certains cas, elles prennent des proportions presque comparables à celles d'un cycle complet de négociations commerciales multilatérales » (OMC, Un commerce ouvert sur l'avenir, p. 64).

16. "China's Accession to the WTO», Focus OMC, octobre 1998, p. 4.

17. "WTO-Übereinkommen Chinas mit den USA. Ruf nach einer umfassenden Liberalisierung», Neue Zürcher Zeitung, 16.11.99; «Les Etats-Unis ouvrent la porte de l'OMC à la Chine populaire», Le Temps, 16.11.99. 
Organisation mondiale du commerce tant que la Chine ne nous avait pas rejoints. La Chine doit encore parvenir à un accord avec les autres gouvernements membres et il nous faut achever d'importantes discussions techniques avant qu'elle puisse prendre la place qui lui revient à la table des grandes nations commerçantes. ${ }^{18}$

Les pays en développement et les pays en transition souhaitent que les négociations en vue de l'accession des autres candidats se terminent bientôt. Une des idées en circulation serait l'organisation d'une session spéciale de négociations intensives, mais la confrontation pour l'élection du nouveau directeur général et les désaccords sur le contenu du nouveau cycle de négociations commerciales multilatérales ont relégué cette idée à l'arrière-plan.

\section{$\square$ Travaux du Conseil général}

Le Conseil général est l'organe de l'OMC chargé d'exécuter les fonctions de l'organisation entre les réunions de la Conférence ministérielle. Il comprend tous les Membres de l'OMC. En 1999, il a donné la priorité à la préparation de la Conférence de Seattle (voir plus loin sous-chapitre 2.3). Il a également supervisé les travaux menés conformément à la Déclaration de Singapour de décembre 1996 concernant les liens entre commerce et investissement, la transparence des marchés publics, la simplification des procédures dans les échanges commerciaux et l'interaction du commerce et de la politique de la concurrence.

Ces questions faisaient également partie de la préparation de la Conférence ministérielle de Seattle. Après l'échec de celle-ci, leur traitement futur paraît incertain. La Suisse attache une importance particulière à l'établissement d'un consensus dans ce domaine ${ }^{19}$.

\section{$\square$ Agriculture}

Le Comité de l'agriculture a suivi l'application des engagements concernant l'accès aux marchés des produits agricoles, la limitation du soutien interne aux agriculteurs et la réduction des subventions à l'exportation. Il a aussi débattu des fonctions non commerciales de l'agriculture, c'est-à-dire de son rôle dans l'économie, dans la protection de l'environnement et dans la politique de sécurité alimentaire. La multifonctionnalité de l'agriculture justifie, pour l'Union européenne, le Japon, la Norvège et la Suisse notamment, des mesures de soutien spécifiques.

L'article 20 de l'Accord sur l'agriculture prévoit l'ouverture de négociations au début de cette année dans le but de poursuivre le processus de libéralisation du secteur agricole. Cet objectif général cache de très fortes divergences.

\section{$\square$ Commerce des services}

L'Accord général sur le commerce des services prévoit, à l'article XIX, que les membres engagent des séries de négociations successives en vue d'élever progressivement le niveau de la libéralisation, cinq ans au plus tard après la création de l'OMC. Ce moment est arrivé. Le Conseil du commerce des services a

18. «Le directeur général se félicite de l'accord sino-américain mais tient à souligner qu'il reste beaucoup à faire avant l'accession de la Chine », Communiqué de presse, n 148, OMC, Genève, 15.11.99.

19. «Activités courantes de l'OMC», Rapport sur la politique économique extérieure 99/1+2, chiffre 422. 
consacré beaucoup de temps à la préparation de cette négociation et a réussi à s'entendre relativement bien sur les points qui auraient dû figurer à ce sujet dans la Déclaration de Seattle.

Le Conseil du commerce des services a aussi discuté du commerce électronique et de l'accord informel de 1998. D’après celui-ci, le commerce électronique n'est pas imposé pour le moment.

\section{$\square$ Propriété intellectuelle}

L'Accord sur les aspects des droits de propriété intellectuelle qui touchent au commerce (Accord sur les ADPIC) prévoit qu'il doit être appliqué début 1996 par les pays développés, début 2000 par les pays en développement (ou en transition) et enfin début 2006 par les pays les moins avancés ${ }^{20}$. Les membres de l'OMC sont tenus de notifier leur législation de mise en œuvre de l'accord dans ces délais. Cependant, beaucoup de pays en développement n'ont pas encore légiféré dans ce domaine et ils ont sollicité des délais supplémentaires.

Le Conseil des ADPIC s'est aussi penché sur d'autres dispositions de l'accord. Le réexamen de l'article 27.3.b concernant la brevetabilité des végétaux et des animaux a fait l'objet de vives controverses.

Au cours de l'année 1999, la Communauté de travail des œuvres d'entraide suisses et la Déclaration de Berne sont intervenues à plusieurs reprises sur ces questions $^{21}$. Elles ont demandé à la Confédération de soutenir les pays qui sollicitent des prolongations de délais pour la mise en œuvre de l'Accord sur les ADPIC. Elles lui demandèrent aussi de se prononcer, dans le débat sur la révision de l'article 27.3.b, en faveur du maintien de la possibilité de protéger les êtres vivants autrement que par des brevets ${ }^{22}$. Dans une lettre datée du 10 septembre, l'ambassadeur Pierre-Louis Girard, délégué du Conseil fédéral aux accords commerciaux (Seco), répondit qu'il était «important de laisser aux pays en développement le temps nécessaire pour mettre en œuvre l'Accord sur les ADPIC dans son état actuel». Concernant l'article 27.3.b, «la Suisse est d'avis que la flexibilité de la solution actuelle doit être conservée » ${ }^{23}$.

\section{$\square$ Règlement des différends}

Le Mécanisme de règlement des différends est de plus en plus sollicité. Le Secrétariat de l'OMC estime qu'il fonctionne de façon efficace ${ }^{24}$. La plupart des différends ont été réglés. Cependant, certains litiges n'ont pas pu être aplanis. Le cas le plus connu concerne le «régime applicable à l'importation, à la vente et à la distribution des bananes », qui oppose les Communautés européennes à quatre pays latino-américains et aux Etats-Unis ${ }^{25}$. En application des décisions

20. Cf. dispositions transitoires prévues par les articles 65 et 66 de l'Accord.

21. N. Keim, "Conséquences du brevet sur la vie pour les pays du Sud», Conférence de presse de la Communauté de travail des œuvres d'entraide suisses, Berne, 6.4.99; «Cinq ans après l'OMC, où en est l'Inde avec les brevets?», Vers un développement solidaire, octobre 1999, pp. 4-11.

22. Lettre adressée le 22 juillet 1999 au Seco, à David Syz, secrétaire d'Etat, et à l'ambassadeur Pierre-Louis Girard, délégué aux accords commerciaux. Les ONG fondaient leur réflexion sur une étude de Philippe Cullet achevée en 1999 au Centre de recherche en droit international de l'environnement (Genève), intitulée «Revision of the TRIPS Agreement concerning the Protection of Plant Varieties: Lessons from India concerning the Development of a Sui Generis System».

23. Lettre adressée à la Déclaration de Berne et à la Communauté de travail.

24. Rapport annuel 1999, vol. 1, p. 4.

25. Ibid., pp. 91-92. 
de l'Organe de règlement des différends (ORD), les Communautés européennes ont révisé le régime d'importation. L'ORD a toutefois estimé que celui-ci restait incompatible avec les règles de l'OMC et il a autorisé les Etats-Unis à revenir sur des concessions commerciales à concurrence du montant du dommage subi.

La Suisse souhaite que la procédure de règlement des différends soit clarifiée et renforcée. Le Mémorandum d'accord devrait par exemple préciser quelle instance est autorisée à décider que les mesures prises par une partie perdante sont satisfaisantes $^{26}$.

\section{Activités de coopération technique}

L'OMC veut aider les pays en développement ou en transition à s'intégrer au système commercial multilatéral et à en bénéficier. Elle contribue au renforcement des capacités institutionnelles et à la formation des ressources humaines afin de favoriser l'expansion commerciale, la participation aux négociations internationales et la mise en œuvre des accords conclus.

Le budget ordinaire de l'OMC destiné à la coopération technique et à la formation s'élève à un peu plus de 2 millions de francs suisses. Des contributions volontaires complètent ce budget. Considérant le caractère permanent de ces activités et les besoins financiers croissants, plusieurs donateurs ont proposé une rationalisation. A titre transitoire, ils ont constitué un Fonds global d'affectation spéciale pour la coopération technique ${ }^{27}$. Celui-ci est ouvert depuis le $1^{\text {er }}$ juillet 1999 à tous les membres de l'OMC. Les donateurs demandent qu'ultérieurement, la coopération technique émarge au budget ordinaire de l'OMC.

L'OMC finance aussi le Centre du commerce international (CCI), qui est une institution du système des Nations Unies chargée de la coopération technique dans le domaine du commerce. Le centre fonctionne sous l'égide de l'OMC et de la CNUCED. Ces deux institutions contribuent à part égale à son budget ordinaire, qui s'est élevé à 28,7 millions de francs suisses en $1998^{28}$. Le centre aide les pays en développement et en transition (également au niveau des entreprises) à exploiter au mieux leur potentiel d'exportation et à améliorer leurs opérations d'importation. Parmi les nombreux projets gérés par le centre, il convient de mentionner le Programme intégré conjoint CCI/CNUCED/OMC d'assistance technique à des pays moins avancés sélectionnés et d'autres pays africains $^{29}$. Ce programme est doté d'un Fonds commun d'affectation spéciale, financé par une dizaine de pays.

\section{$\square$ L'appui de la Suisse}

Le Secrétariat d'Etat à l'économie (Seco) dépense environ 20 millions de francs par an pour la coopération technique liée au commerce, dont 5 millions pour le renforcement des politiques commerciales ${ }^{30}$.

26. «Clarification et renforcement de la procédure de règlement des différends », Rapport sur la politique économique extérieure $99 / 1+2$, chiffre 141 .

27. Danemark, Finlande, Pays-Bas, Norvège, Suède, Suisse, Royaume-Uni, Communautés européennes et Hongkong/Chine. Cf. OMC, Rapport annuel 1999, vol. 1, p. 114.

28. Ibid., p. 123 .

29. Ibid., pp. 114-115. Le programme bénéficie à huit pays: Bénin, Burkina Faso, Ouganda, Tanzanie (pays classés parmi les moins avancés), Côte d'Ivoire, Ghana et Kenya (pays à faible revenu), ainsi que Tunisie (pays à revenu intermédiaire).

30. «Mesures de soutien aux pays en développement ou en transition», Rapport sur la politique économique extérieure 99/1+2, chiffre 52; Memorandum: Swiss Contribution for Technical Assistance on Trade Policy (mimeo), Seco, Bern, 21.10.99, 2 p. 
La participation suisse au Programme intégré conjoint CCI/CNUCED/OMC d'assistance technique mérite d'être relevée, car elle favorise un regroupement de forces. Pour la période 1998-2001, les contributions suisses s'élèvent à 2 millions pour le Ghana et à 1 million pour l'Ouganda. Concernant le renforcement des politiques commerciales des pays en développement ou en transition, le Seco distingue les appuis donnés à Genève et les actions réalisées dans les pays bénéficiaires ${ }^{31}$.

A Genève, le Seco finance notamment:

- l'Agence de coopération et d'information pour le commerce international (ACICI). Il s'agit d'un nouveau service qui fait partie de la structure d'accueil de la Genève internationale. Les services de l'agence sont destinés en priorité aux pays les moins favorisés ${ }^{32}$;

- des contributions volontaires aux programmes de coopération technique de

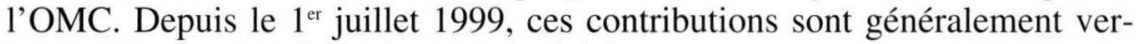
sées au Fonds global d'affectation spéciale;

- la participation de fonctionnaires en provenance de pays en développement ou en transition à des programmes de l'Institut universitaire de hautes études internationales (IUHEI) et de l'Académie de droit international économique et de règlement des différends de l'Université de Genève;

- divers appuis fournis par exemple pour la préparation de la $3^{\text {e }}$ Conférence ministérielle de l'OMC.

Dans les pays bénéficiaires, le Seco intervient sur demande. Il finance notamment des expertises et des actions de formation au Vietnam dans le cadre de la procédure d'accession à l'OMC.

Le Seco finance aussi des organisations non gouvernementales. Le principal bénéficiaire de cet appui est actuellement le Centre international pour le commerce et le développement durable (CICDD) à Genève ${ }^{33}$. Le Seco estime que ce centre fait un «excellent travail» en diffusant l'information sur les politiques commerciales au sein de la société civile et en nouant des contacts entre les milieux commerciaux et les $\mathrm{ON}^{34}$.

\section{$\square$ Environnement et développement durable}

Les Accords de l'OMC ne comprennent pas de texte portant spécifiquement sur l'environnement et sur le développement durable. Cependant, plusieurs accords contiennent des dispositions à ce sujet. En avril 1994, la Réunion ministérielle de Marrakech a créé le Comité du commerce et de l'environnement (CCE). Elle lui a confié un double mandat: «identifier les relations entre les mesures commerciales et les mesures environnementales de manière à promouvoir le développement durable» et «faire des recommandations appropriées pour déterminer s'il y a lieu de modifier les dispositions du système commercial multilatéral, en en respectant le caractère ouvert, équitable et non discriminatoire ${ }^{35}$.

31. Ibid.

32. Site Internet www.acici.org.

33. International Centre for Trade and Sustainable Development (ICTSD), site Internet www.ictsd.org.

34. Memorandum: Swiss Contribution for Technical Assistance on Trade Policy (miméo), Seco, Bern, 21.10.99, p. 2.

35. «Décision sur le commerce et l'environnement», Résultats des négociations commerciales multilatérales du Cycle d'Uruguay. Textes juridiques, GATT, Genève, 1994, p. 494. 
En 1999, le CCE a poursuivi l'étude des effets de la libéralisation des échanges dans les secteurs de l'agriculture, de la pêche, de la sylviculture, du cuir, de l'énergie et des services environnementaux. Des participants ont rappelé que les subventions faussent les échanges et maintiennent souvent des modes de production périmés qui nuisent à l'environnement ${ }^{36}$. Le CCE a aussi rencontré les représentants de cinq secrétariats d'accords environnementaux multilatérau ${ }^{37}$.

Afin de favoriser des synergies, l'OMC et l'ONU ont conclu un «arrangement global» qui définit des «éléments de coopération entre l'OMC et le PNUE » ${ }^{38}$. Cet accord favorisera l'échange d'informations, la collaboration aux niveaux de la coopération technique et de la recherche, ainsi que la représentation réciproque aux réunions des deux organisations.

Dans le cadre des mesures prises pour améliorer le dialogue avec la société civile, l'OMC a organisé deux symposiums de haut niveau en mars 1999, successivement sur les thèmes "commerce et environnement» et "commerce et développement». Trois groupes de travail ont traité des questions suivantes dans le cadre du premier symposium:

- liens entre les politiques commerciales et les politiques environnementales;

- synergies entre libéralisation des échanges, protection de l'environnement, croissance économique soutenue et développement durable;

๖ interaction entre les milieux commerciaux et les milieux s'occupant de protection de l'environnement ${ }^{39}$.

Enfin, l'OMC a publié en octobre 1999 un rapport de la division de la recherche et des analyses économiques, intitulé L'environnement et le commerce ${ }^{40}$. Ce rapport traite des dimensions économiques et politiques de l'interface entre le commerce et l'environnement. Il affirme que les politiques commerciales et environnementales ne sont pas forcément contradictoires: elles peuvent au contraire se renforcer mutuellement.

Le Conseil fédéral estime que les Accords de l'OMC doivent mieux tenir compte des principes généraux du droit international de l'environnement. Il donne l'exemple du «principe de précaution», qui permet d'interdire la commercialisation d'un produit lorsque celui-ci présente une menace de dommage irréversible, même en l'absence de certitude scientifique. Le Conseil fédéral souhaite aussi que l'OMC accepte l'application systématique des principes du «pollueur-payeur» et de la durabilité. D'autre part, l'OMC doit admettre que les paiements directs aux agriculteurs et les taxes d'incitation contribuent à la protection de l'environnement. Toujours dans le même but, il faudrait différencier les biens selon les méthodes de production employées ${ }^{41}$. L'information des consommateurs grâce à la création de labels et à l'étiquetage des produits per-

36. «Comité du commerce et de l'environnement ", in OMC, Rapport annuel 1999, vol. 1, pp. 110-112; OMC, Rapport $d u$ Comité du commerce et de l'environnement 1999; idem, Bulletin sur le commerce et l'environnement, $n^{\circ s} 28$ à 31 . Voir aussi Le commerce et l'environnement dans le cadre de l'OMC, disponible sur le site Internet www.wto.org/wto/ environ/environm.htm.

37. «Members Discuss WTO's Relationship with Environmental agreements», WTO Focus, May-June 1999, pp. 6-7.

38. «Eléments de coopération entre l'OMC et le PNUE», Communiqué de presse, OMC, Genève, 29.11.99.

39. «Symposium de haut niveau sur le commerce et l'environnement », Focus OMC, mars 1999, pp. 3-5.

40. H. Nordström and S. Vaughan, Trade and Environment, Special Studies no 4, WTO, Geneva, October 1999, 110 p. ; aperçu dans Focus OMC, septembre-octobre 1999, pp. 1, 7-10, et dans L'Agefi, 5.10.99.

41. "Prise en compte des aspects de la protection de l'environnement », Rapport sur la politique économique extérieure $99 / 1+2$, chiffre 144 
met de faire un pas dans cette direction. Il faudra toutefois éviter que ces méthodes de commercialisation ne servent à limiter l'accès aux marchés ${ }^{42}$.

\subsection{CONFÉRENCE MINISTÉRIELLE DE L'OMC - (SEATTLE, 30.11-3.12.1999)}

Dans la structure de l'OMC, l'autorité suprême est la Conférence ministérielle, composée de représentants de tous les membres de l'organisation. La conférence se réunit au moins une fois tous les deux ans. Elle peut prendre des décisions sur toutes les questions relatives aux accords commerciaux multilatéraux.

Les Accords actuels de l'OMC sont issus des négociations du Cycle d'Uruguay de 1986-1994. Ce résultat s'est traduit par une vaste réforme des règles du commerce mondial et par la réduction des obstacles au commerce. Les participants ont toutefois admis que ce processus devait se poursuivre. Ils ont donc prévu le lancement de nouvelles négociations, notamment sur l'agriculture, les services et certains aspects des droits de propriété intellectuelle liés au commerce.

En 1996, la $1^{\text {re }}$ Conférence ministérielle de l'OMC, réunie à Singapour, a en outre entrepris un programme de travail sur les investissements, la concurrence, la transparence des marchés publics et la facilitation des échanges. Sur l'insistance des pays en développement, la $2^{\mathrm{e}}$ Conférence ministérielle de Genève a décidé en mai 1998 d'évaluer la mise en œuvre des accords existants et la réalisation de leurs objectifs. Elle a chargé le Conseil général, organe exécutif de l'OMC, de présenter à la Conférence ministérielle de Seattle un programme de nouvelles négociations commerciales multilatérales. Des recommandations concernant l'organisation devaient garantir que le programme de travail soit «commencé et achevé rapidement ${ }^{43}$.

\section{$\square$ Préparation de la conférence}

Les premières propositions sur les thèmes de la négociation ont été présentées en mars 1999. En septembre, le Conseil général a commencé la rédaction du projet de Déclaration ministérielle, qui devait être publiée à l'issue de la Conférence de Seattle. Ce projet était fondé sur plus de 150 propositions des pays membres.

Fin octobre 1999, à un mois de la conférence, les membres de l'OMC n'avaient pas beaucoup progressé dans la recherche d'un consensus sur les objectifs du nouveau cycle de négociations commerciales multilatérales. Long d'une trentaine de pages, le document préparatoire de la Conférence de Seattle restait une compilation laborieuse des différentes propositions. De multiples parenthèses marquaient des divergences souvent inconciliables.

Pour éviter un échec de la conférence, les représentants de 25 membres influents $^{44}$ de l'OMC se sont réunis informellement à Lausanne les 25 et 26 octobre, sous la présidence du conseiller fédéral Pascal Couchepin. A son arrivée, il déclara: "Il n'est pas impossible qu'à Seattle les Etats membres de l'OMC n'arrivent pas à s'entendre et que l'on décide de se revoir. $»^{45}$ Après

\footnotetext{
42. Contribution de la délégation suisse pour le Symposium OMC de haut niveau sur le commerce et l'environnement (miméo), Seco, Berne, 15.3.99, 5 p.

43. OMC, Rapport annuel 1999, vol. 1, p. 5

44. Dont les Etats-Unis, l’Union européenne et le Japon du côté des pays industrialisés; l’Inde, l'Egypte, le Brésil et la Thaỉlande du côté des pays en développement.

45. Le Temps, 26.10.99.
} 
avoir mis leurs divergences sur la table, les participants ont toutefois fait des déclarations conciliantes avant de se séparer. La Communauté de travail des œuvres d'entraide suisses regretta l'absence des pays les moins avancés (PMA) à la rencontre de Lausanne ${ }^{46}$.

Afin de favoriser la participation de ces derniers, le Secrétariat de l'OMC organisa une semaine d'information à Genève début novembre 1999. La Norvège, la Grande-Bretagne et la Suisse financèrent les déplacements et les séjours des représentants de 37 pays membres et observateurs de l'OMC qui ne disposent pas d'un bureau permanent à Genève ${ }^{47}$.

Quelques jours avant la Conférence, les négociateurs tentèrent pour une dernière fois d'aplanir leurs divergences - en vain ${ }^{48}$.

\section{$\square$ Ouverture de la conférence et organisation des travaux}

L'ouverture de la conférence fut retardée par des manifestations populaires. Le 30 novembre, dès l'aube, des milliers d'opposants à la conférence se déployèrent autour du Centre des congrès de l'Etat de Washington, formant de véritables barrières humaines pour empêcher les délégués de prendre part à la séance d'ouverture. Des mouvements aux objectifs souvent très divers, mais unis dans la lutte contre l'ultralibéralisme incarné, à leurs yeux, par l'OMC. «Aujourd'hui, tout le monde était dans la rue, en provenance des pays riches comme des pays pauvres, écologistes, activistes des droits de l'homme, syndicalistes, paysans et défenseurs des droits sociaux », déclara le leader paysan français José Bové 49 .

Le $1^{\text {er }}$ décembre, les ministres ont engagé des négociations sur le projet de déclaration élaboré au cours des mois précédents par le Conseil général. Ils ont créé des groupes de travail ouverts à toutes les délégations, et devaient travailler presque en continu jusqu'à la clôture de la Conférence, le 3 décembre.

La conférence était placée sous la présidence de Charlene Barshefsky, représentante pour les questions commerciales internationales des Etats-Unis, le pays hôte. Elle a déploré les «actions irresponsables d'une toute petite minorité », qui avaient retardé l'ouverture. Concernant le déroulement de la conférence, elle a annoncé que pour favoriser l'obtention du consensus sur certains points, elle organiserait des réunions avec un nombre restreint de délégations dans le cadre du «salon vert». Cette décision a mécontenté les pays ainsi exclus d'une partie des négociations tandis que d'autres estimaient que les différents groupes d'intérêts, y compris les PMA, étaient équitablement représentés. Enfin, Charlene Barshefsky a indiqué que même si un groupe de travail parvenait à s'entendre sur un texte, aucun élément de la Déclaration ministérielle ne serait approuvé tant que tous ne le seraient pas.

46. Agence Télégraphique Suisse, citée par La Côte (Nyon), 27.10.99.

47. «L'OMC organise une "semaine de Genève" pour les délégations sans représentation", Communiqué de presse, $\mathrm{n}^{\circ}$ 141, OMC, Genève, 26.10.99.

48. «Peut-on encore sauver Seattle?», Tribune de Genève, 25.11.99; «Der Weg nach Seattle ist sehr steinig. Ergebnislose Vorbereitungsarbeiten bei der WTO in Genf », Neue Zürcher Zeitung, 25.11.99.

49. Le Temps, 1.12.99. 


\section{Groupes de travail et principaux thèmes de discussion}

\section{Agriculture}

Principaux points du projet de texte:

- application renforcée des règles de l'OMC à l'agriculture;

- accès aux marchés;

- nouvelles réductions des subventions à l'exportation;

- diminution du soutien interne;

๑ préoccupations autres que commerciales et multifonctionnalité;

- questions relatives aux pays en développement.

Principaux désaccords:

- Le commerce des produits agricoles doit-il être intégralement soumis, à terme, aux mêmes règles que le commerce des autres produits?

- Les subventions à l'exportation doivent-elles disparaître entièrement?

- Des pays pourront-ils continuer à protéger leur agriculture en invoquant ses fonctions non commerciales (environnement, sécurité alimentaire)?

\section{Mise en œuvre et règles}

Demandes des pays en développement:

- des délais pour la mise en œuvre des Accords concernant les aspects des droits de propriété intellectuelle qui touchent au commerce (ADPIC), les Mesures concernant les investissements et liées au commerce (MIC), ainsi que l'évaluation en douane:

- une modification de certaines dispositions de l'Accord antidumping, de l'Accord sur les subventions et de l'Accord sur les textiles:

- des engagements fermes de la part des pays industrialisés concernant le traitement spécial et différencié des pays en développement;

- une prolongation du traitement préférentiel que I'Union européenne accorde aux pays d'Afrique, des Caraïbes et du Pacifique (ACP), afin de laisser à ces 71 pays le temps de s'intégrer dans l'économie mondiale.

\section{Accès aux marchés}

Principaux dossiers en suspens:

- champ et portée des négociations: doivent-elles couvrir l'ensemble des produits non agricoles?

- définition d'un objectif global concernant les réductions tarifaires;

- mesures non tarifaires affectant l'accès aux marchés;

- exonération générale par les pays industrialisés des droits de douane sur leurs importations en provenance des pays les moins avancés.

\section{Programme de Singapour (1996) et autres questions}

Points discutés par le groupe:

• établissement de nouvelles règles concernant les investissements;

- politique de la concurrence;

- transparence des marchés publics;

๑ facilitation des échanges.

\section{Questions systémiques}

Points discutés par le groupe:

- structure de décision de l'OMC:

- amélioration de la transparence;

๑ échanges d'informations;

๑ diffusion des documents;

๖ participation du public. 


\section{Commerce et normes du travail}

Ce groupe a été constitué le 2 décembre seulement afin d'examiner les propositions de certains pays industrialisés concernant la discussion des normes du travail (au sein de l'OMC ou d'un organe administré conjointement par plusieurs organisations internationales). Beaucoup de pays en développement se sont opposés à cette initiative.

Source: "Résumé des réunions, 1", 2 et 3 décembre 1999", Note d'information de l'OMC (disponible sur le site Internet de l'OMC www.wto.org)

\section{$\square$ Groupes d'intérêts}

Les coalitions se font et se défont souvent en fonction des thèmes en discussion. La plupart des analystes estiment néanmoins que quatre alliances ont joué un rôle prépondérant à Seattle $\mathrm{e}^{50}$ :

- les Etats-Unis donnent la priorité à l'agriculture ainsi qu'aux services. Ils s'attaquent au protectionnisme agricole de l'Union européenne et du Japon (ainsi que de la Suisse). La poursuite de la libéralisation de ces deux secteurs ayant été décidée à la fin du Cycle d'Uruguay en $1994^{51}$, leur position est forte;

- les pays exportateurs de produits agricoles non subventionnés («Groupe de Cairns ») cherchent à accroître leurs ventes sur les marchés protégés d'Europe, d'Amérique et du Japon. Le groupe comprend des pays développés (Canada, Australie, Nouvelle-Zélande) et en développement (notamment Argentine, Brésil, Chili, Uruguay, Thaïlande, Malaisie, Philippines, Indonésie et Afrique du Sud). Il s'est allié au Etats-Unis sur la question de l'ouverture des marchés agricoles;

- l'Union européenne est à l'origine de la proposition d'entreprendre le «Cycle du millénaire». Elle souhaite un éventail de négociations large (de même que la Suisse, la Norvège, le Japon et la Corée du Sud), comprenant la libéralisation des investissements, la politique de la concurrence, la transparence des marchés publics et la facilitation des échanges, conformément au programme de travail décidé à Singapour en 1996. Elle espère, grâce à la négociation simultanée de différents dossiers, obtenir des concessions dans le domaine agricole et faire accepter le principe de la multifonctionnalité de ce secteur;

- les pays en développement insistent sur l'application, par les pays industrialisés, des règles concernant l'accès aux marchés. Ils exigent un renforcement rapide de l'assistance technique liée au commerce ${ }^{52}$. L'acceptation de ces revendications constitue à leur avis un préalable au lancement d'une nouvelle série de négociations. Ils sollicitent des délais supplémentaires pour la mise en œuvre de différents accords (mentionnés dans l'encadré ci-dessus). Les pays en développement s'opposent généralement à l'adoption de normes sociales et environnementales susceptibles de favoriser les politiques néoprotectionnistes du Nord.

50. «Sommet de l'OMC à Seattle: mode d'emploi », Le Temps, 29.11.99, pp. 2-3.

51. Cf. Accord sur l'agriculture, article 20, et Accord général sur le commerce des services, article XIX.

52. Cf., dans le sous-chapitre 2.2, «Activités de coopération technique». 


\section{$\square$ Position de la Suisse}

Dans ses prises de position, la Confédération a tenté de promouvoir «un programme de négociation suffisamment large pour couvrir les intérêts et les besoins de tous ${ }^{53}$. Aucun sujet ne devait être écarté a priori. Cette approche pragmatique devait permettre de mieux équilibrer les concessions et les acquis. Par contre, un programme de négociation partielle d'accès au marché, dans les seuls domaines de l'agriculture et des services, risquait, aux yeux des négociateurs suisses, de ne pas donner de résultats significatifs. Comme nous l'avons constaté plus haut, la Suisse est solidaire sur ce point des pays qui protègent fortement leur agriculture. Des observateurs favorables aux positions du Groupe de Cairns et des Etats-Unis ont qualifié cette approche de «tout sauf l'agriculture», accusant l'Union européenne et ses alliés de vouloir éloigner l'attention de ce dossier épineux ${ }^{54}$.

La Suisse souhaitait que les points suivants figurent au programme du cycle de négociations ${ }^{55}$ :

- poursuivre la libéralisation du commerce des biens et des services, ainsi que des marchés publics. Tous les pays développés et certains pays émergents devraient procéder à de nouvelles réductions des tarifs douaniers et continuer à démanteler les barrières non tarifaires ;

- améliorer l'accès au marché dans le domaine des services grâce à la levée des obstacles et à l'harmonisation des législations nationales ;

口 instaurer des règles multilatérales dans les domaines de la concurrence et des investissements privés ;

- concernant l'agriculture, tenir compte des aspects non commerciaux tels que la sécurité alimentaire ou la protection de l'environnement. Ces objectifs sont également vitaux pour les pays en développement ${ }^{56}$;

口 renforcer la coopération technique commerciale avec les pays à faible revenu;

- supprimer progressivement les préférences tarifaires dont bénéficient encore les pays en développement les plus avancés;

- favoriser la cohérence entre les politiques commerciales et environnementales (en assurant notamment la cohérence entre les dispositions commerciales des accords internationaux sur l'environnement et les règles de l'OMC);

- renforcer la coopération entre l'OMC et l'OIT concernant les normes de travail fondamentales;

$\checkmark$ accroître la transparence du système commercial multilatéral et intensifier le dialogue avec la société civile.

53. P.-L. Girard, «Les nouvelles négociations OMC: quel programme de négociation?», La Vie économique, novembre 1999, p. 7.

54. «Sommet de l'OMC à Seattle: mode d'emploi», Le Temps, 29.11.99, p. 2.

55. P. Couchepin, «Discours devant le Club suisse de la presse, le 5 novembre 1999 » (miméo), Seco, Berne; idem, «Déclaration à la Conférence ministérielle, Seattle, 30 novembre 1999», OMC, Genève, WT/MIN(99)/ST/2; P.-L. Girard, «Les nouvelles négociations OMC: quel programme de négociation?», La Vie économique, novembre 1999, pp. 7-9.

56. P.-L. Girard, «Les nouvelles négociations OMC: quel programme de négociation? », La Vie économique, novembre 1999, p. 8. 


\section{$\square$ Une meilleure coordination entre les pays du Sud}

La position des pays en développement s'est renforcée grâce à des réflexions et à des prises de position communes. Concernant la Conférence de Seattle, deux types d'action méritent d'être relevés:

- le Centre $S u d^{57}$ a réalisé différentes études sur la place des pays en développement au sein du système commercial multilatéral. En décembre 1998, il a publié le livre The WTO Multilateral Trade Agenda and the South, qui distingue trois domaines d'action pour le tiers monde: la mise en œuvre des Accords du Cycle d'Uruguay, les négociations sur l'agriculture et les services prévues dans ces accords (le fameux "agenda incorporé»), et les nouveaux enjeux. Jusqu'en 1998, les pays développés ont toujours réussi, selon les auteurs du livre, à imposer leurs objectifs de négociation. Désormais, le Sud doit faire valoir ses revendications: réduire la marginalisation des pays les moins avancés, libéraliser non seulement les mouvements de biens, de services et de capitaux, mais aussi les migrations des travailleurs, mettre le système commercial multilatéral au service du développement et, enfin, faire appliquer effectivement l'égalité des droits des membres de l'OMC. Le Centre Sud estime que la transparence et les «décisions par consensus » sont indispensables à la réalisation de ces objectifs;

口 au cours de sa $9^{\mathrm{e}}$ rencontre ministérielle à Marrakech, en septembre 1999, le Groupe des $77^{58}$ a exigé que les Accords de 1'OMC soient réévalués, corrigés et réformés. Relayée par les sites Internet des organisations non gouvernementales, l'expression anglaise reviewed, repaired and reformed (RRR) a instantanément fait le tour du monde.

\section{$\square$ Interventions des organisations non gouvernementales (ONG)}

Les médias ont surtout relaté les manifestations de masse organisées par les ONG à Seattle et à travers le monde. En réalité, elles mènent une action en profondeur depuis des années. Les ONG sont innombrables et ont des objectifs très divers. Les plus connues sont notamment (citées dans l'ordre alphabétique):

- Friends of the Earth, Londres;

- Institute for Agriculture and Trade Policy (IATP), Minneapolis (Etats-Unis);

- International Centre for Trade and sustainable Development (ICTSD), Genève ;

\ International Coalition for Development Action, Bruxelles;

- Peoples' Global Action (PGA);

- Public Citizen Global Trade Watch, Washington DC;

๖ Third World Network, Penang (Malaisie);

๖ WWF International, Gland (Suisse).

Les ONG s'opposent à des négociations visant essentiellement la libéralisation et l'expansion des échanges mondiaux (migrations non comprises). Elles

57. Le Centre Sud (The South Centre), établi à Genève et à Dar es-Salaam, est une organisation intergouvernementale permanente des pays en développement depuis 1995. Il inspire le Mouvement des pays non alignés et le Groupe des 77 par ses recherches et ses publications.

58. Le Groupe des 77 a vu le jour lors de la création de la CNUCED en 1964 et compte actuellement plus de 130 membres. 
exigent un changement radical de direction afin de promouvoir un commerce équitable et le développement durable. Par conséquent, les nouvelles négociations commerciales multilatérales doivent être un «cycle du développement»ou ne pas avoir lieu.

\section{$\square$ Position des ONG suisses}

Les organisations qui cherchent à influencer la politique suisse de développement suivent depuis longtemps les activités du GATT, devenu l'OMC. Il s'agit plus particulièrement de la Déclaration de Berne/Association pour un développement solidaire et de la Communauté de travail (CT), qui regroupe les œuvres d'entraide Swissaid, Action de carême, Pain pour le prochain, Helvetas et Caritas. En 1999, leurs prises de position et interventions concernant le nouvel ordre commercial mondial ont été plus nombreuses que jamais. L'encadré de la page suivante en illustre quelques moments marquants.

\section{$\square$ L'échec de la conférence}

Les trois jours de débats n'ont pas permis de réduire les désaccords de façon significative. Les participants à la conférence ont par conséquent décidé de «marquer une pause ». Le directeur général de l'OMC, Mike Moore, déclara, peu après son retour de Seattle, qu'il allait engager des consultations avec les délégations et étudier de nouveaux moyens de réduire les écarts et d'améliorer le processus de négociation, afin de «pouvoir dire au plus vite aux ministres que nous sommes prêts à reconvoquer la Conférence ministérielle et à la mener à bien ${ }^{59}$.

Un ensemble complexe de causes explique l'échec de la conférence ${ }^{60}$. On peut distinguer trois types:

• l'Union européenne et ses alliés avaient le plus intérêt à l'ouverture d'un nouveau cycle de négociations, s'il comprenait un ensemble de thèmes intégré. D'autres pays pouvaient se contenter d'un programme restreint. Les Etats-Unis et le Groupe de Cairns n'avaient pas grand-chose à perdre en cas d'échec car l'ouverture de pourparlers sur les échanges de produits agricoles et de services était de toute façon fixée pour l'an 2000. Ces pays n'avaient donc pas besoin de faire de concessions importantes pour atteindre un de leurs principaux objectifs. De même, les pays en développement exigeaient l'application des accords existants avant d'en conclure de nouveaux. Ils ont bénéficié sur ce point de l'appui des ONG;

- les désaccords qui persistaient à l'ouverture de la conférence étaient beaucoup trop profonds pour être aplanis en quatre jours. La règle du consensus exigeait que toutes les parties donnent leur accord, ce qui impliquait que chacune y trouve son compte. Les manifestations, la visite du président Clinton et le défaut d'organisation ont fortement réduit le temps dont les négociateurs pouvaient effectivement disposer;

59. «Il est vital de maintenir et de consolider ce que nous avons acquis», Communiqué de presse, $\mathrm{n}^{\circ}$ 160, OMC, Genève, 7.12.99.

60. Il existe beaucoup d'explications. Voir par exemple «The Aftermath of Seattle: A Summary of Views», BRIDGES Weekly Trade News Digest (bridges@iatp.org), vol. 3, n 48, 15.12.99; S. George, «Seattle: le tournant. Comment l'OMC fut mise en échec", Le Monde diplomatique, janvier 2000, pp. 4-5; N. Keim, «WTO Summit in Seattle: Victory for Some, Defeat for Others?», Swiss Coalition News, no 22, January 2000, pp. 12-14; P. Niggli, «PostSeattle: Whither Now? ", Swiss Coalition News, n 22, January 2000, pp. 12-14; S. Oetliker, «WTO: Jetzt heisst es handeln und weiter verhandeln», Cash, 10.12.99, pp. 78-79; «Préparation d'un nouveau cycle de négociations sur le commerce mondial », Rapport sur la politique économique extérieure 99/1+2, chiffre 421 . 
Avril

Conférence de presse de la CT

Lettre de la CT à Pascal Couchepin

Mai

Conférence de presse de la CT
Conséquences du brevet sur la vie pour les pays du Sud.

Les pays du Sud doivent rester libres d'adopter des démarches autonomes différentes. II faut donc réviser l'Accord sur les aspects des droits de propriété intellectuelle qui touchent au commerce (ADPIC).

Position de la CT concernant les relations entre le commerce, le développement et l'environnement.

Demande d'inclure un représentant de la CT dans la délégation suisse à Seattle. (Cette demande n'a pas abouti mais la délégation a régulièrement rencontré les $0 N G$.)

\section{Août}

Déclaration de Manifeste des organisations non gouvernementales suisses.

Berne, Pro Natura, Lancer un cycle d'évaluation et de réflexion au lieu d'un nouveau cycle pour la libéUnion suisse des paysans, Fondation pour la protection des consommateurs

Nouveau cycle de libéralisation à I'OMC:

d'abord tenez les anciennes promesses et réglez les questions en suspens!

Le nouveau cycle menace l'économie et l'agriculture des pays défavorisés du Sud. La révision de l'Accord sur l'agriculture doit permettre à ces pays de protéger leur développement agricole.

ralisation. Propositions:

๑ évaluer les conséquences économiques,

sociales et écologiques des accords précédents;

$\checkmark$ respecter les fonctions sociales

et environnementales de l'agriculture (multifonctionnalité);

a mieux intégrer le Sud dans le marché mondial;

a protéger l'environnement;

$\checkmark$ respecter les droits sociaux;

ـ favoriser la transparence et la participation.

Octobre

Lettre de la CT au Position suisse à la $3^{\circ}$ Conférence ministérielle de I'OMC.

Conseil fédéral Pour un véritable Round du développement.

Refuser d'étendre les négociations à de nouveaux domaines, tels que les investissements.

Satisfaire en priorité les revendications du Sud.

Novembre

Organisations

Déclaration commune: "Pas de chèque en blanc pour l'administration."

syndicales,

paysannes,

écologiques, CT

Nous demandons que I'OMC examine les répercussions de ses règles actuelles (review), qu'elle en corrige les retombées négatives (repair) et qu'elle entreprenne une réforme des règles commerciales dans l'intérêt des populations et de l'environnement (reform). Nous refusons les «nouveaux thèmes" du Millenium Round.

Sources: documentations de presse et informations de la Communauté de travail des œuvres d'entraide suisses. Site Internet www.swisscoalition.ch.

• les marges de manœuvre étaient faibles. Les Etats-Unis étaient déjà en campagne électorale et ne pouvaient pas accepter un programme de négociations touchant des domaines sensibles (mesures antidumping et textiles notamment). L'Union européenne, le Japon et la Suisse acceptaient bien l'inscription de l'agriculture au programme, mais ne prévoyaient pas de faire des concessions majeures par la suite. Dans ces conditions, le Groupe de Cairns n'avait pas grand-chose à gagner dans un nouveau cycle. Quant aux pays en 
développement qui avaient déjà ouvert unilatéralement leurs économies à partir des années 80 dans le cadre des politiques d'ajustement structurel, ils attendaient que les pays du Nord jouent réellement le jeu de la libéralisation.

L'échec de la Conférence de Seattle n'empêchera pas l'ouverture des négociations sectorielles prévues, mais celles-ci ne bénéficieront pas au départ de l'insertion dans un cycle étendu. Le 7 février 2000, le Conseil général de l'OMC a fixé le début des négociations sur les services à fin février et sur l'agriculture au 23 mars $^{61}$.

\subsection{CONFÉRENCE DES NATIONS UNIES SUR LE COMMERCE ET LE DÉVELOPPEMENT (CNUCED)}

Créée en 1964 en tant que mécanisme intergouvernemental permanent, la CNUCED est le principal organe de l'ONU dans le domaine du commerce et du développement. Elle aide les pays du tiers monde à tirer le meilleur parti des possibilités de commerce, d'investissement et de développement qui s'offrent à eux, à faire face aux problèmes découlant de la mondialisation et à s'intégrer de façon équitable dans l'économie mondiale. Elle se réunit tous les quatre ans sur un autre continent ${ }^{62}$.

La CNUCED compte actuellement 188 Etats membres. Ses fonctionnaires (environ 400) font partie du Secrétariat de l'ONU. Le budget annuel de fonctionnement s'élève à 50 millions de dollars. Les activités de coopération technique chiffrées à 24 millions de dollars sont financées par des ressources extrabudgétaires ${ }^{63}$.

Lors de sa $9^{\mathrm{e}}$ session à Midrand (Afrique du Sud) en 1996, le mandat de l'organisation a été redéfini et elle a entrepris des réformes importantes. Cette démarche a été un succès, si l'on se réfère aux commentaires appréciatifs lors de la reconduction du secrétaire général Rubens Ricupero dans ses fonctions en février $1999^{64}$. De nombreux pays ont manifesté leur satisfaction quant à l'efficacité accrue de la CNUCED. Parmi les nouveaux points forts de l'organisation, les intervenants ont mentionné ${ }^{65}$ :

- le nouvel esprit de coopération régnant entre les Etats membres depuis 1996;

- la qualité du travail analytique de la CNUCED;

\ sa contribution au développement des pays les moins avancés;

- l'aide apportée aux économies en transition dans des domaines tels que la dette extérieure et les négociations commerciales;

61. «WTO "Back in Business" with Agriculture and services», BRIDGES Weekly Trade News Digest, vol. 4, n 5, 8.2.00.

62. CNUCED, Un partenariat pour la croissance et le développement, CNUCED, Service des relations extérieures, Genève, 1998, p. 1.

63. Ibid. Mis à jour en décembre 1999, site Internet www.unctad.org.

64. «Le secrétaire général de la CNUCED est reconduit dans ses fonctions. M. Ricupero mènera l'organisation vers le XXI" siècle ", Communiqué de presse, CNUCED, Genève, 19.2.99.

65. Ibid. Le communiqué résume notamment les interventions du Groupe des 77, de la Chine, de l'Union européenne, de la Fédération de Russie, du Japon et des Etats-Unis. 
- la qualité du travail accompli dans la préparation des pays en développement à la Conférence ministérielle de l'OMC à Seattle ${ }^{66}$;

ـ sa collaboration avec la société civile et le secteur privé.

ASTM 1997, sur la redéfinition du mandat et les réformes institutionnelles de la CNUCED (pp. 37-43).

Ca ASTM 1998, idem (pp. 182-183).

\section{$\square$ Préparation de la 10 session de la CNUCED (Bangkok, 12-19 février 2000)}

Réuni en session exécutive le 5 février 1999, le Conseil du commerce et du développement, l'organe directeur de la CNUCED, a approuvé l'ordre du jour de la $10^{\mathrm{e}}$ session de la conférence. Ce texte constate que la mondialisation a eu «des effets contrastés» et n'a pas réduit les disparités économiques entre les pays. Il ajoute que «des tensions et des déséquilibres de nature systémique sont également apparus et, compte tenu du degré élevé d'interdépendance, le risque de bouleversements touchant par effet de contagion de nombreux pays et régions a considérablement augmenté». La communauté internationale est donc invitée à «entreprendre un examen rigoureux et équilibré du cadre directif et institutionnel du système commercial et financier mondial $»^{67}$.

L'examen comprendra aussi la question de l'intégration des pays en développement et en transition dans l'économie mondiale. L'objectif consiste à identifier les stratégies susceptibles de faire de la mondialisation un instrument efficace du développement.

Selon Carlos Fortin, secrétaire général adjoint, la $10^{\mathrm{e}}$ session permet d'élargir le concept d'intégration de la société civile dans le travail de l'organisation car celle-ci a fait appel à des acteurs extérieurs tels qu'instituts de recherche, syndicats et $\mathrm{ONG}$ pour un "processus de réflexion collective ${ }^{68}$.

Cette approche s'apparente aux travaux du Sommet Partenaires pour le développement (Lyon, 9-12 novembre 1998). Elle suscite un débat sur le choix, la légitimité et la représentativité des partenaires. La question se pose aussi des conséquences éventuelles en matière de concurrence (avantages discriminatoires) d'une collaboration avec le secteur privé. Il s'agit donc de définir un code de conduite.

Le 25 octobre 1999, le Conseil du commerce et du développement a commencé les négociations du Plan d'action de Bangkok. Le Maroc a présenté la proposition du Groupe des 77, suite à la réunion préparatoire des pays en développement tenue à Marrakech en septembre. Le groupe réaffirme que «la CNUCED doit jouer un rôle clé en incitant la communauté internationale à mettre en place les mesures et les initiatives identifiées dans le Plan d'action afin d'assurer l'intégration des pays en développement dans l'économie mondiale sur une base équitable. Ces mesures ont trait au commerce international, à la finance et aux investissements ». La CNUCED est «la mieux placée pour forger un consensus en vue de reformuler les politiques» de développement. Elle a un rôle majeur à jouer dans la conception d'une «intégration échelonnée dans l'économie mondiale » ${ }^{69}$.

66. Cf. «La politique commerciale internationale en 1999 et au-delà», Rapport sur le commerce et le développement 1999 , pp. 44-48.

67. «En route pour Bangkok: le Conseil adopte l'ordre du jour de la $10^{\circ}$ session de la CNUCED», Communiqué de presse, CNUCED, Genève, 5.2.99.

68. Ibid.

69. «La communauté internationale entame des négociations sur la prochaine CNUCED», Communiqué de presse, CNUCED, Genève, 25.10.99. 
$\mathrm{Au}$ nom de l'organisation non gouvernementale Third World Network, Ch. Raghavan a déclaré que «les économies néolibérales et le consensus de Washington n'ont pas répondu aux attentes, la mondialisation non plus. Les économistes [...] commencent à réclamer de la flexibilité pour les pays en développement en ce qui concerne les règles et disciplines et un traitement préférentiel et plus favorable jusqu'à ce que ces pays parviennent à un niveau de développement qui leur permette d'assumer davantage d'obligations $\gg^{70}$.

Dans son rapport à la CNUCED X, Rubens Ricupero recommande, «au-delà de l'unification des marchés», la construction d'une "communauté mondiale de coopération et de connaissance partagée pour la sécurité et le développement». Après avoir rappelé «les causes de l'impasse actuelle», le rapport propose «un nouvel engagement international en faveur de la croissance et du développement» en améliorant «la conduite d'une économie internationale en voie de mondialisation». Il indique comment la CNUCED, «institution fondée sur le savoir au service du développement», peut accroître sa contribution grâce aux partenariats et à la recherche d'une plus grande efficacité ${ }^{71}$.

En janvier 2000, le Seco a annoncé les objectifs de la participation de la Suisse à la 10e session de la CNUCED: «A Bangkok, la Suisse s'engagera pour que la CNUCED X reste un forum ouvert et critique pour toutes les questions relatives au commerce, à l'investissement et au développement. L'intégration des pays en développement et des pays en transition doit, comme jusqu'à présent, rester l'objectif premier de cette organisation. A l'avenir, les activités de recherche et d'analyse ainsi que l'assistance technique devront dûment tenir compte des discussions tenues à l'OMC et des impératifs liés au développement durable. La Suisse insistera également pour une plus grande transparence et une meilleure politique d'information du secrétariat de la CNUCED. ${ }^{72}$

\section{$\square$ Commerce, développement durable et genre}

A la $9^{e}$ session de la CNUCED, il avait été décidé que les activités liées à la prise de responsabilité (empowerment) et à la démarginalisation des femmes seraient traitées comme un thème transversal.

Dans le cadre du processus préparatoire de la CNUCED X, un atelier d'experts s'est réuni à Genève les 12 et 13 juillet 1999. Il avait pour thème "Commerce, développement durable et sexospécificité ${ }^{73}$. Les experts ont analysé les impacts très complexes de la mondialisation en matière de discrimination sexuelle. De nombreux cas révèlent une réduction des inégalités de revenu et des disparités femmes-hommes; d'autres montrent au contraire une aggravation.

L'industrie manufacturière a massivement fait appel à la main-d'œuvre féminine. Celle-ci a ainsi participé davantage à l'économie monétarisée, mais au moment de la récession consécutive à la crise financière asiatique, un nombre disproportionné de femmes ont perdu leur emploi. Les femmes constituent toujours la majorité des pauvres en milieu rural. Quand l'expansion du commerce mondial a provoqué une hausse de la production des cultures d'exportation, la main-

70. Ibid.

71. Rapport du secrétaire général de la CNUCED à la 10 CNUCED, Genève, Nations Unies, 1999.

72. «Participation de la Suisse à la CNUCED X», Communiqué de presse, Seco, Berne, 19.1.00.

73. «Le développement passe par la démarginalisation des femmes», Note aux correspondants, $\mathrm{n}^{\circ} 24$, CNUCED, Genève, 15.7.99. 
d'œuvre féminine a été davantage mobilisée, mais sa rétribution n'a pas augmenté proportionnellement. Dans le secteur commercial, les femmes des pays les plus pauvres sont particulièrement discriminées. Très présentes dans le petit commerce, elles sont inexistantes dans les transactions internationales. La globalisation a aussi stimulé l'émigration des femmes à la recherche de nouvelles opportunités économiques. Cependant, les coûts sociaux des migrations sont élevés.

Suite à ces observations, les experts ont élaboré une série de recommandations. La CNUCED a publié les travaux de l'atelier en octobre $1999^{74}$. La Conférence de Bangkok donnera l'occasion de mettre en évidence les enjeux clés concernant la sexospécificité et d'introduire cette dimension dans le Plan d'action quadriennal de la CNUCED.

\section{SOURCES}

CNUCED, Rapport sur le commerce et le développement 1999, Nations Unies, New York et Genève, octobre 1999.

Conseil fédéral, «Rapport sur la politique économique extérieure», Feuille fédérale, annuel.

Cullet, Ph., «Revision of the TRIPS Agreement concerning the Protection of Plant Varieties: Lessons from India concerning the Development of a Sui Generis System», The Journal of World Intellectual Property, vol. 2, no 4, July 1999, pp. 617-656.

GATT, Résultats des négociations commerciales multilatérales du Cycle d'Uruguay. Textes juridiques, GATT, Genève, 1994.

George, S., «Seattle: le tournant. Comment l'OMC fut mise en échec», Le Monde diplomatique, janvier 2000, pp. 4-5.

Girard, P.-L., «Les nouvelles négociations OMC: quel programme de négociation?», La Vie économique - Revue de politique économique, novembre 1999, pp. 6-9.

IATP, BRIDGES Weekly Trade News Digest (bridges@iatp.org), diffusé par l'Institute of Agriculture and Trade Policy (IATP), Minneapolis (Etats-Unis).

ICTSD/CICDD, Bridges between Trade and Sustainable Development (mensuel), International Centre for Trade and Sustainable Development (ICTSD)/Centre International pour le Commerce et le Développement Durable (CICDD), Genève.

Keim, N., «WTO Summit in Seattle: Victory for Some, Defeat for Others?», Swiss Coalition News, ${ }^{\circ} 22, J^{2}$ anuary 2000 , pp. 12-13.

Niggli, P., «Post-Seattle: Whither Now?», Swiss Coalition News, n 22, January 2000, pp. 13-14.

Oetliker, S., «WTO: Jetzt heisst es handeln und weiter verhandeln», Cash, 10.12.99, pp. 78-79.

$\mathrm{OMC}$, Bulletin sur le commerce et l'environnement, OMC, Genève.

OMC, Focus OMC. Bulletin d'information (mensuel), OMC, Genève.

OMC, Rapport annuel 1999 (2 vol.), OMC, Genève.

OMC, Rapport du Comité du commerce et de l'environnement (annuel), OMC, Genève.

OMC, Un commerce ouvert sur l'avenir ( $2^{c}$ édition révisée), OMC, Genève, juin 1999.

South Centre, The WTO Multilateral Trade Agenda and the South, The South Centre, Geneva, 1998.

UNCTAD, Trade, Sustainable Development and Gender, papers prepared in support of the themes discussed at the PreUNCTAD X Expert Workshop on Trade, Sustainable Development and Gender (Geneva, 12-13 July 1999), United Nations, New York and Geneva, 1999.

\section{SITES INTERNET}

ACICI: www.acici.org.

CICDD: www.ictsd.org.

CNUCED: www.unctad.org.

OMC: www.wto.org.

Seco: www.seco-admin.ch.

ADRESSES UTILES

Agence de coopération et d'information pour le commerce international (ACICI), 9, rue de Varembé, case postale 156, 1211 Genève 20; tél. 022/910.31,50, fax 022/910.31.51.

Centre international pour le commerce et le développement durable (CICDD)/International Centre for Trade and Sustainable Development (ICTSD), 13, chemin des Anémones, 1219 Genève; tél. 022/917.84.92, fax 022/917.80.93.

Conférence des Nations Unies sur le commerce et le développement (CNUCED), Palais des Nations, 8-14, avenue de la Paix, 1211 Genève 10; tél. 022/907.12.34, 022/917.12.34, fax 022/907.00.43.

Organisation mondiale du commerce (OMC), Centre William Rappard, 154, rue de Lausanne, 1211 Genève 21 ; tél. 022/739.51.11, fax 022/739.54.58.

Secrétariat d'Etat à l'économie (Seco), Palais fédéral Est, 3003 Berne; tél. 031/322.56.56, fax 031/322.56.00.

74. UNCTAD, Trade, Sustainable Development and Gender, United Nations, New York and Geneva, 1999. Les recommandations figurent aux pages 447-451 de la publication. 